\title{
MAXIMIZING STUDENT LEARNING OUTCOMES WHEN USING BUSINESS Strategy Simulation ToOls: The Role of Pedagogy, PARTICIPATION, AND PRACTICE (A Multi-YeAR StUdy)
}

\author{
Kevin A. Fletcher
}

The BRC Academy Journal of Business 11, no. 1 (2021): 163-194

https://dx.doi.org/10.15239/j.brcacadjb.2020.11.01.ja07

\section{WEB APPENDIX}

https://dx.doi.org/10.15239/j.brcacadjb.2021.11.01.wa07 


\section{APPENDIX \#1 - Summary of Capsim Decisions Made by Students}

In the Capsim Capstone simulation, students (individuals or teams) make decisions to help company performance in the following specific ways (Capsim Professor Guide, 2018):

- $R \& D$ - Students have the ability to make changes to each of their existing products for the coming round/fiscal year. Customers in each market segment demand faster, smaller sensors each year, but the rate of change for that demand is different for each market segment. So, the size and speed of the sensor can be modified along with mean time before failure (MTBF). Each of these changes also changes the perceived age of the products. Likewise, each of the product characteristics is deemed important in the customer buying decision, but at varying rates of importance. So, students must balance performance demands for each market segment, with the accumulating cost of the changes and the time it takes for R\&D to launch those product changes. Significant changes in the product characteristics or launching a new product might mean that product is not available for many months to even more than a year after the changes are ordered and increases costs.

- Production - In the production area, students must take the forecasted sales amount (from marketing) and order production with enough units to meet monthly demand. This includes hiring up to two full shifts worth of staff, investing in automation (which can reduce labor costs long-term), and even ordering new plant capacity to be built as the market segment growth eventually outpaces the capacity for each company and the entire industry.

- Marketing - In the marketing area of the simulation, students forecast product demand for the coming year, set prices, and budget for marketing and sales of the products. 
- Finance - Once students make decisions, they must finance those decisions. They have the option to issue stock, borrow money (short term debt) or issue bonds (long term debt). They can also buy back stock, issue dividends, and set their accounts payable and receivable (AP/AR) policies (i.e., 30 day, 60 day).

The following additional, optional decision-making areas further enhance and complicate the simulation environment as such (Capsim Professor Guide, 2018):

- Total Quality Management (TQM) - Additional investments can be made in one or more of ten areas designed to increase the quality of the production process and products. Options such as Quality Initiative Training or Six Sigma Training have various effects and impacts on R\&D cost and production time as well as production costs. Students have full access to those projected impacts in advance.

- Advanced Marketing - In lieu of budgeting a bulk amount on marketing and sales, students can control how to spend that money through various channels (i.e., radio ads, trade shows). Each market segment and corresponding customer base responds differently to different types of marketing and sales investments. Likewise, investments in the number and type of sales staff can be made when this part of the simulation is activated.

- Labor Negotiations - Labor negotiates with companies in the industry to determine the most favorable set of wages and benefits - thus affecting those costs for all companies in the industry. Potential wage increases and even worker strikes can affect the entire industry and individual companies, respectively.

- Human Resources - Additional decisions to spend more on recruiting and staff training are made with this option - each with associated direct costs and benefits (i.e., more efficient staff leading to lower labor costs per unit of production). 


\begin{tabular}{|c|c|c|c|c|c|c|c|}
\hline & Team A & Team B & Team C & Team D & Team E & Team F & $\begin{array}{l}\text { Student } \\
\text { Teams } \\
\text { Average } \\
\text { Scores }\end{array}$ \\
\hline Class $1^{\mathrm{A}}$ & $\begin{array}{l}1223 \\
(85)[41] \\
\{21.3\}\end{array}$ & $\begin{array}{l}1045 \\
(113)[64] \\
\{28.3\}\end{array}$ & $\begin{array}{l}1341 \\
(86)[48] \\
\{21.5\}\end{array}$ & $6437^{*}$ & $5696^{*}$ & $6109^{*}$ & 1203 \\
\hline Class 2 & $\begin{array}{l}3906 \\
(162)[49] \\
\{32.4\}\end{array}$ & $\begin{array}{l}3085 \\
(228)[75] \\
\{45.6\}\end{array}$ & $\begin{array}{l}3916 \\
(212)[78] \\
\{42.4\}\end{array}$ & $\begin{array}{l}1178 \\
(176)[68] \\
\{35.2\}\end{array}$ & $6475^{*}$ & $6287^{*}$ & 3021 \\
\hline Class $3^{\mathrm{NM}}$ & $\begin{array}{l}5183 \\
(271)[69] \\
\{38.7\}\end{array}$ & $\begin{array}{l}5213 \\
(295)[65] \\
\{42.1\}\end{array}$ & $\begin{array}{l}1773 \\
(216)[46] \\
\{30.9\}\end{array}$ & $\begin{array}{l}4918 \\
(404)[89] \\
\{50.5\}\end{array}$ & $\begin{array}{l}2660 \\
(197)[48] \\
\{28.1\}\end{array}$ & $6259^{*}$ & 3949 \\
\hline Class 4 & $\begin{array}{l}2189 \\
(184)[71] \\
\{36.8\}\end{array}$ & $\begin{array}{l}3876 \quad(221) \\
{[59]\{44.2\}}\end{array}$ & $\begin{array}{l}5192 \\
(469)[165] \\
\{93.8\}\end{array}$ & $\begin{array}{l}3331 \\
(273)[97] \\
\{45.5\}\end{array}$ & $6293^{*}$ & $6515^{*}$ & 3647 \\
\hline${\operatorname{Class~} 5^{\mathbf{N}}}$ & $\begin{array}{l}4578 \\
(483)[113] \\
\{80.5\}\end{array}$ & $\begin{array}{l}5705 \\
(321)[85] \\
\{53.5\}\end{array}$ & $\begin{array}{l}2712 \\
(412)[119] \\
\{68.7\}\end{array}$ & $\begin{array}{l}3577 \\
(243)[47] \\
\{34.7\}\end{array}$ & $\begin{array}{l}3969 \\
(282)[103] \\
\{47\}\end{array}$ & $6365^{*}$ & 4108 \\
\hline Class 6 & $\begin{array}{l}3178 \\
(333)[103] \\
\{83.3\}\end{array}$ & $\begin{array}{l}3478 \\
(357)[105] \\
\{71.4\}\end{array}$ & $\begin{array}{l}3342 \\
(220)[57] \\
\{44\}\end{array}$ & $\begin{array}{l}3070 \\
(240)[61] \\
\{48\}\end{array}$ & $6038^{*}$ & $6359 *$ & 3267 \\
\hline Class $7^{\mathrm{N}}$ & $\begin{array}{l}1240 \\
(168)[62] \\
\{42\}\end{array}$ & $\begin{array}{l}3268 \\
(134)[45] \\
\{33.5\}\end{array}$ & $\begin{array}{l}4675 \\
(210)[91] \\
\{52.5\}\end{array}$ & $6367^{*}$ & $6027 *$ & $6201 *$ & 3061 \\
\hline
\end{tabular}




\begin{tabular}{|l|l|l|l|l|l|l|l|}
\hline Class 8 $^{\text {GN }}$ & 3631 & 3459 & 5565 & 5470 & 3462 & $5624^{*}$ & 4317 \\
& $(332)[62]$ & $(611)[232]$ & $(472)[135]$ & $(574)[175]$ & $(340)[96]$ & & \\
& $\{55.3\}$ & $\{101.8\}$ & $\{78.7\}$ & $\{95.7\}$ & $\{56.7\}$ & & \\
\hline
\end{tabular}

Table \#1: Summary Capsim Analyst Report Scores for "Non-Footrace" Student Groups (Before Pedagogical Change)

Average student-run company scores (33 total): 3467

Average computer-run company scores (15 total): 6203

* Denotes computer-run companies

A - Six rounds run, adjusted to represent eight

$\mathrm{M}-$ Mixed MBA and Undergraduate teams

$\mathrm{N}$ - Night class (once a week)

$\mathrm{G}$ - Graduate-level class (MBA)

$(\mathrm{x})$ - indicates the total number of log-ins for the team

$[\mathrm{x}]$ - indicates the number of log-ins from individual on the team who logged in the most

$\{\mathrm{x}\}$ - indicates the average number of $\log$-ins for each team (total/number of team members)

Table \#2: Summary Capsim Analyst Report Scores for "Footrace" Student Groups (After Pedagogical Change)

\begin{tabular}{|l|l|l|l|l|l|l|l|}
\hline & Team A & Team B & Team C & Team D & Team E & Team & Student \\
& & & & & & F & Teams \\
\hline Class 9 & 6282 & 5280 & 3831 & 863 & 5612 & $6371^{*}$ & 4374 \\
& $\begin{array}{l}(295)[113] \\
\{49.2\}\end{array}$ & $\begin{array}{l}(281)[63] \\
\{5.2\}\end{array}$ & $\begin{array}{l}(231)[73] \\
\{46.2\}\end{array}$ & $\begin{array}{l}(354)[117] \\
(325)[79]\end{array}$ & & \\
\hline Class 10 & 1147 & 2648 & 2517 & $6715^{*}$ & $6157^{*}$ & $6370^{*}$ & 2104 \\
& $(182)[128]$ & $(102)[42]$ & $(114)[49]$ & & & & \\
& $\{60.7\}$ & $\{34\}$ & $\{38\}$ & & & & \\
\hline
\end{tabular}




\begin{tabular}{|l|l|l|l|l|l|l|l|}
\hline Class & 6190 & 3735 & 3586 & $6199^{*}$ & $5807^{*}$ & $5472^{*}$ & 4504 \\
$\mathbf{1 1}^{\text {NG }}$ & $(209)[136]$ & $(214)[88]$ & $(222)[69]$ & & & & \\
& $\{69.7\}$ & $\{53.5\}$ & $\{55.5\}$ & & & & \\
& & & & & & & \\
\hline
\end{tabular}

Average student-run company scores (11 total): 3792

Average computer-run company scores (7 total): 6156

* Denotes computer-run companies

$\mathrm{N}$ - Night class (once a week)

$\mathrm{G}$ - Graduate-level class (MBA)

(x) - indicates the total number of log-ins for the team

$[\mathrm{x}]$ - indicates the number of log-ins from individual on the team who logged in the most

Table \#3: Summary data of Analyst Report Score by Class Type

\begin{tabular}{|l|l|l|l|l|l|}
\hline & Number & Average- & Average & Average Scores & Average Scores \\
& of Teams & Overall & Comparative & Pre- & Post- \\
& in Study & Analyst & Ranking based & Pedagogical & Pedagogical \\
Report & on Analyst & Change & Change \\
(\# Teams) & (\# Teams) \\
\hline Graduate & 8 & 4386 & 15.25 & $4317(5)$ & $4500(3)$ \\
\hline Mixed & 5 & 3949 & 19.8 & 3949 & N/A \\
\hline Undergraduate- & 20 & 3261 & 24.75 & $2785(15)$ & $4375(5)$ \\
\hline Undergraduate- & 11 & 2053 & 24.9 & $3716(8)$ & $2104(3)$ \\
\hline Night & & & & & \\
\hline
\end{tabular}

* Teams were ranked from 1 to 44 based on their Analyst Report scores and then an average of those scores by class type was calculated. 
Table \#4 - Correlations of Average Team Login, Top Login, and Capsim Score

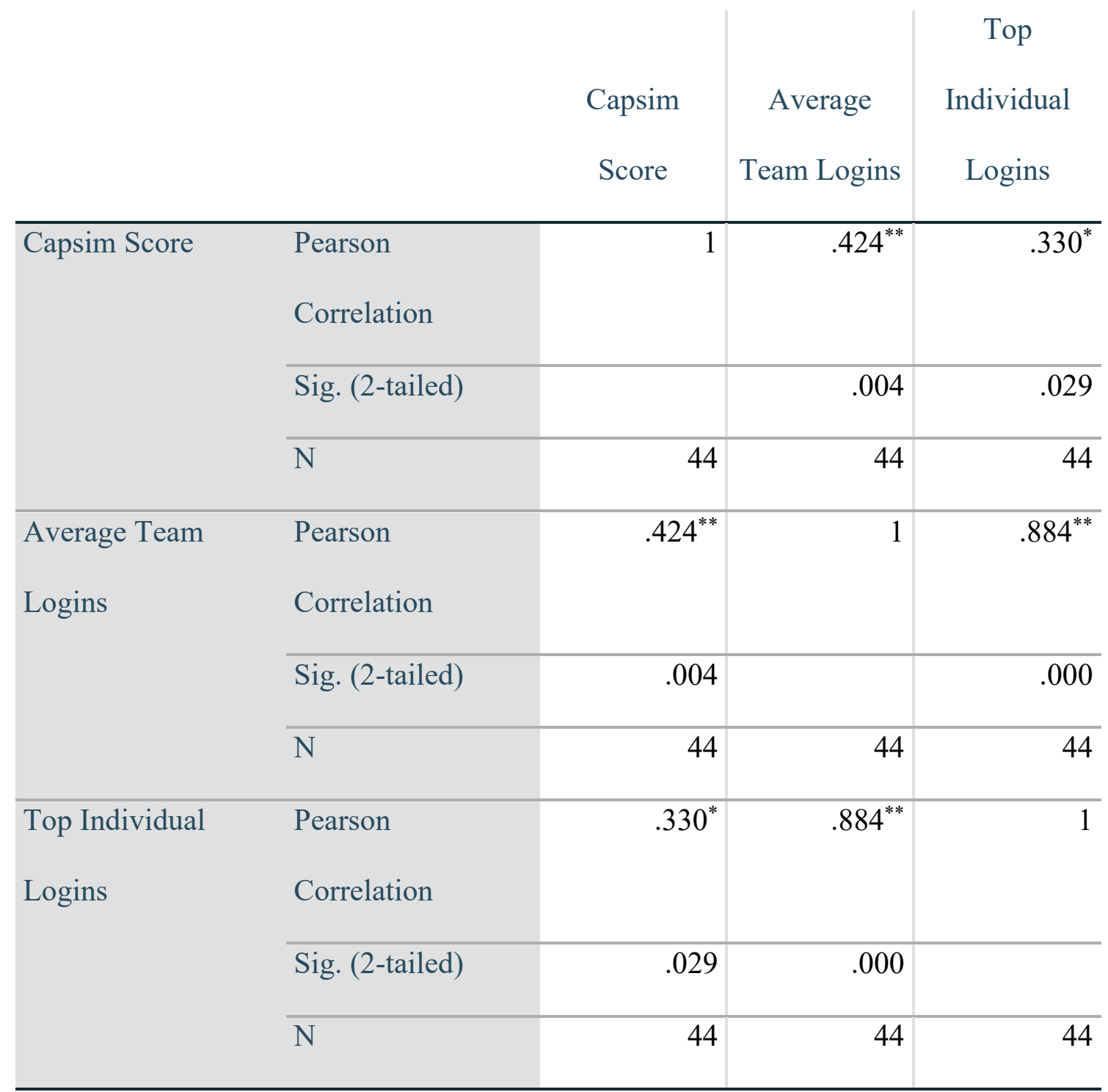

**. Correlation is significant at the 0.01 level (2-tailed).

*. Correlation is significant at the 0.05 level (2-tailed). 
Graph \#1 - Scatterplot-Individual Capsim Scores by Class (Time)

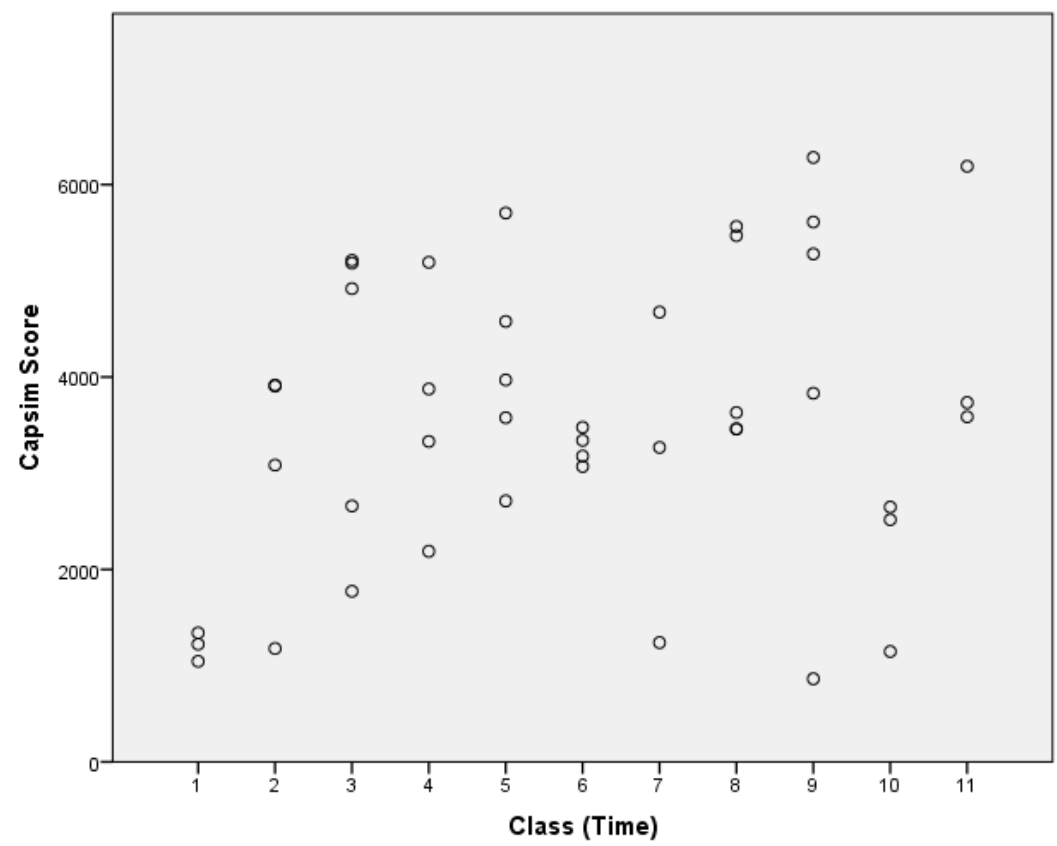

Table \#5: Differences of Average Class Analyst Report Scores Based on Numbers of Student Teams

\begin{tabular}{|l|l|l|l|}
\hline & 3 Student Teams & 4 Student Teams & 5 Student Teams \\
\hline & $(1) 1203$ & $(2) 3021$ & $(3) 3949$ \\
\hline & $(7) 3061$ & $(4) 3647$ & $(5) 4108$ \\
\hline $\begin{array}{l}\text { Average Scores: } \\
\text { Average Scores } \\
\text { without post- }\end{array}$ & $2718) 2104$ & $(6) 3267$ & $(8) 4317$ \\
pedagogical change & $(11) 4504$ & 3311 & $(9) 4374$ \\
groups: & & 3311 & 4124 \\
\hline
\end{tabular}




\begin{tabular}{|l|l|l|l|}
\hline $\begin{array}{l}\text { Average Scores for } \\
\text { just Undergraduate }\end{array}$ & 1203 & 3311 & 4374 \\
Day Teams (20 & (175\% increase from & (32\% increase from \\
teams) & $3)$ & $4)$ \\
\hline
\end{tabular}

(\#) - Indicates Class group number 